\title{
Influence of Dispersive Long Range Interactions on Properties of Vapour-Liquid Equilibria and Interfaces of Binary Lennard-Jones Mixtures
}

\author{
Simon Stephan ${ }^{\mathrm{a}}$ and Hans Hasse ${ }^{\mathrm{a}}$ \\ ${ }^{a}$ Laboratory of Engineering Thermodynamics (LTD), TU Kaiserslautern, 67663 Kaiserslautern, Germany
}

\section{ARTICLE HISTORY}

Compiled December 5, 2019

\begin{abstract}
The influence of dispersive long-range interactions on properties of vapour-liquid equilibria and interfaces of six binary Lennard-Jones (LJ) mixtures was studied by molecular dynamics (MD) simulations and density gradient theory (DGT). The mixtures were investigated at a constant temperature $T$, at which the low-boiling component, which is the same in all mixtures, is subcritical. Two different highboiling components were considered: one is subcritical, the other is supercritical at $T$. Furthermore, the unlike dispersive interaction was varied such that mixtures with three different types of phase behaviour were obtained: ideal, low-boiling azeotrope, and high-boiling azeotrope. In a first series of simulations, the full LJ potential was used to describe these mixtures. To assess the influence of the long-range interactions, these results were compared with simulations carried out with the LJ truncated and shifted (LJTS) potential applying the corresponding states principle. The dispersive long-range interactions have a significant influence on the surface tension and the interfacial thickness of the studied mixtures, whereas the relative adsorption and the enrichment are hardly affected. Furthermore, the influence of the long-range interactions on Henry's law constants and the phase envelopes of the vapour-liquid equilibrium was investigated. The long-range interactions have practically no influence on the composition dependency of the investigated mixture properties.
\end{abstract}

\section{KEYWORDS}

Lennard-Jones fluid; molecular simulation; long-range interaction; interfacial properties; equation of state

\section{Introduction}

In molecular simulations of classical force fields, in which interactions are computed from pairpotentials [1-3], this is done explicitly only up to a certain distance of the interacting sites. If no 
correction is applied, this causes a deviation of the simulation results from what would be obtained with the full potential. Different corrections schemes have been developed to account for the influence of the long-range interactions in simulations [1, 4, 5]. They are based on simplifying assumptions regarding the structure of the fluid. For simple cases, such as in studies of the equilibrium of homogeneous fluids, simple correction terms yield excellent results. In other cases, such as in studies of inhomogeneous fluids with different phases, accounting for the long range interactions is more tedious[6-8], and it is not uncommon to simply truncate the potential in these cases and to neglect the long-range terms[9-11]. More generally, the ways in which long-range interactions are treated in molecular simulations vary largely and it is interesting to evaluate, which influence they have on the simulation results.

This has been studied in great detail for the Lennard-Jones (LJ) 12-6 pair potential [5-8, 1231]. In these studies, the full LJ potential (as approximated by using a large value of the cut-off radius $r_{\mathrm{c}}$ and suitable long-range corrections) is typically compared to either a truncated LJ potential (LJT potential; with some given $r_{\mathrm{c}}$ and without applying long-range corrections), or to the so-called truncated-and-shifted LJ potential (LJTS potential, which is a LJT potential that is shifted in such way that the potential energy becomes zero at $r_{\mathrm{c}}$ [12-15]). In homogeneous systems, the intermolecular forces that are calculated from the LJT and the LJTS potential are the same, if the same $r_{\mathrm{c}}$ is used in both cases, whereas the energy and the pressure are affected by the shifting.

Different observables show different sensitivities to long-range interactions: The influence of the truncation has been studied for virial coefficients [25], the vapour-liquid equilibrium (VLE) [13, 15, 16], and vapour-liquid interfacial properties $[18,21]$. The influence of the long-range interactions on phase equilibria and properties of inhomogeneous systems has been of particular interest [17-23, 26, 29, 31$37]$.

To the best of our knowledge, all previous studies regarding the influence of the long-range interactions refer to pure fluids only, and no study has been performed yet on the influence of the long-range interactions for mixtures. We have therefore carried out an investigation of the influence of these interactions on binary vapour-liquid interfacial properties and the corresponding phase equilibria of binary fluid mixtures by comparing results of the LJ and LJTS potential.

Vapour-liquid interfaces of mixtures are important in many fields of science and engineering. They have recently received particular attention as in many systems an enrichment of components at this interface occurs, which is thought to have an influence on the mass transfer [38-43]. Vapour-liquid interfacial properties of Lennard-Jones mixtures have been investigated many times in the literature by molecular simulations, density gradient theory (DGT) or density functional theory [39, 41, 44-50]. Reports on studies of the influence of the size and energy parameters of the pure components as well 
as the influence of the cross interactions on binary interfacial properties are available [41, 46, 47]. Also results on the composition- and temperature-dependency of the enrichment of components at these interfaces have been published [39, 41, 44, 45].

In the present work, the influence of the dispersive long-range interactions on VLE properties (isothermal phase envelopes and Henry's law constants) and interfacial properties (surface tension, interfacial thickness, relative adsorption, and enrichment) of binary LJ and LJTS mixtures were investigated. The MD results for the interfacial properties of the LJTS mixtures were taken from an earlier work of our group [41]. The computer experiments were accompanied by calculations with equations of state (EOS) and density gradient theory. An equation of state for the LJ fluid [51] and the LJTS fluid [52] (PeTS EOS) from the literature were used for the DGT and for the computation of phase equilibria.

\section{Modelling and simulation}

\subsection{Model potential}

The Lennard-Jones (LJ) potential is defined as the pairwise-additive and spherically symmetric potential

$$
u_{\mathrm{LJ}}(r)=4 \varepsilon\left[\left(\frac{\sigma}{r}\right)^{12}-\left(\frac{\sigma}{r}\right)^{6}\right]
$$

where $r$ is the distance between two particles. The parameters $\sigma$ and $\varepsilon$ characterize the diameter of the particles and the strength of the attractive interaction, respectively. Simulations of the LJ potential were performed with a truncated potential in combination with a correction of long-range interactions $[1,53]$. The interactions were explicitly computed to a cut-off radius of $5 \sigma$ in the present work and long-range interactions beyond were considered with an implicit correction scheme, taking into account inhomogeneities in simulations of two-phase systems as described in more detail below. The corresponding results are referred to as 'results of the LJ potential' in the following.

For comparison, the Lennard-Jones truncated and shifted potential (LJTS) is used. The truncation radius of the LJTS potential is $2.5 \sigma$ throughout the present work:

$$
u_{\mathrm{LJTS}}(r)= \begin{cases}u_{\mathrm{LJ}}(r)-u_{\mathrm{LJ}}\left(r_{\mathrm{c}}\right) & r \leq 2.5 \sigma \\ 0 & r>2.5 \sigma\end{cases}
$$

The corresponding results are referred to as 'results of the LJTS potential'. 
The interaction parameters $\varepsilon$ and $\sigma$ were chosen to be the same for the binary mixtures modelled by the LJ and the LJTS potential. The cross interactions of the mixtures were described by the modified Lorentz-Berthelot combination rules [54, 55]:

$$
\begin{aligned}
& \sigma_{12}=\frac{\sigma_{1}+\sigma_{2}}{2}, \\
& \varepsilon_{12}=\xi_{12} \sqrt{\varepsilon_{1} \varepsilon_{2}},
\end{aligned}
$$

where $\xi_{12}$ is a state-independent binary interaction parameter.

\subsection{Binary mixtures}

Six binary mixtures (labelled A - F) were investigated, cf. Fig. 1. For all binary mixtures, the highboiling component is labelled as '1' and the low-boiling component as '2'. Each mixture was investigated using the LJ potential and the LJTS potential. The temperatures in this pair of simulations was chosen such that the reduced temperature with respect to the critical temperature of the high-boiling component $T_{\text {red }}=T / T_{\mathrm{c}, 1}$ was the same for both LJ and LJTS potential. This results in $T=0.92 \varepsilon k_{B}^{-1}$ for the LJ mixtures and $T=0.77 \varepsilon k_{B}^{-1}$ for the LJTS mixtures, which corresponds in both cases to approximately $T / T_{\mathrm{c}, 1}=0.7$. The critical temperature of the pure LJ and LJTS fluid has been reported numerous times in the literature, cf. Refs. $[53,56,57]$ for recent reviews of such data. Note, that the reported values show significant scattering and thus are only known within a certain confidence interval. Table 1 summarizes critical and triple point data of the LJ and LJTS fluid that are used in the present work with estimated uncertainties.

The size parameter and the mass of both components are the same for all six mixtures A - F, i.e. $\sigma_{1}=\sigma_{2}$ and $M_{1}=M_{2}$ respectively. The vapour pressure of the low-boiling component 2 was decreased by decreasing the dispersion energy compared to component 1. Two different low-boiling components were considered: for the first, the ratio of dispersion energies was $\varepsilon_{2} / \varepsilon_{1}=0.9$; for the second it was $\varepsilon_{2} / \varepsilon_{1}=0.5$. Also the binary interaction parameter was varied. Three values were chosen $\xi_{12}=1,1.2$ and 0.85 . These variations result in six mixtures $(\mathrm{A}-\mathrm{F})$ that were investigated. Their phase behaviour is shown qualitatively in Fig. 1 . Depending on the choice of $\varepsilon_{2} / \varepsilon_{1}$, component 2 is either subcritical (mixtures A - C) or supercritical (mixtures D - F). For $\xi_{12}=1$ the mixture is almost ideal in the sense of Raoult's law, the value of $\xi_{12}=1.2$ causes a negative deviation from Raoult's law and $\xi_{12}=0.85$ causes positive deviation from Raoult's law. This leads to a high-boiling azeotrope for mixture B and a low-boiling azeotrope for the mixture C.

All physical properties are reported in the present work with respect to the Lennard-Jones poten- 
tial parameters [1] of the high-boiling component $\varepsilon_{1}, \sigma_{1}$ and the molecular mass $M$, as well as the Boltzmann constant $k_{B}$.

\subsection{Molecular simulations}

\section{Direct vapour-liquid equilibrium simulations}

The simulations for the coexisting vapour and liquid phase were carried out in the $N V T$ ensemble with 16,000 particles using the MD code $l s 1$ [58]. A liquid film was positioned in a rectangular box such that two planar vapour-liquid interfaces were oriented perpendicular to the $z$-axis. The time step was $\Delta t=0.001 \sigma \sqrt{M / \varepsilon}$. The equilibration was executed for 2,000,000 time steps. For the initialization, the saturated densities and the bulk phase compositions of the mixtures were estimated with the corresponding EOS $[51,52]$. The production was carried out for 6,000,000 time steps. Density and pressure profiles were calculated in block averages of 500,000 time steps during the production phase. The statistical error was estimated to be three times the standard deviation of all block averages. The elongation of the simulation box normal to the interface was $80 \sigma$ and the thickness of the liquid film in the centre of the simulation box was $40 \sigma$. The elongation in the directions parallel to the interface was at least $20 \sigma$. The pressure and density profiles were calculated in $z$-direction at 1200 bins. A slab-based long-range correction scheme based on the approach of Janeček [6, 7] was used for the LJ potential simulations.

The saturated densities $\rho^{\prime}$ and $\rho^{\prime \prime}$, the vapour pressure $p$, and the saturated vapour and liquid phase composition $x_{i}^{\prime}$ and $x_{i}^{\prime \prime}$ were calculated as an average over the respective phases excluding the area close to the interface. Also the density profiles along the $z$-axis were obtained as averages over all sampled block averages.

The interfacial tension was calculated via the mechanical route, i.e. from the deviation between the normal and the tangential diagonal components of the overall pressure tensor $[59,60]$

$$
\gamma=\frac{1}{2} \int_{-\infty}^{\infty}\left(p_{\mathrm{N}}-p_{\mathrm{T}}\right) \mathrm{d} z
$$

The applied simulation procedure is described in detail in Ref. [41].

\section{Henry's law constant simulations}

Henry's law constants $H_{2,1}$ of the low-boiling component 2 in component 1 were sampled in the $N p T$ ensemble with 1372 solvent particles using the MD code $m s 2$ [61]. The Henry's law constants were obtained from the residual chemical potential of the solute at infinite dilution from the relation [62]

$$
H_{2,1}=\rho^{\prime} T \exp \left(\mu_{2}^{\infty} / T\right),
$$


where $\rho^{\prime}$ is the saturated liquid density of the solvent and $T$ is the temperature. The residual chemical potential at infinite dilution $\mu_{2}^{\infty}$ was computed using Widom's test particle method [63]. The equilibration was carried out for 300,000 time steps and the production for 1,000,000 time steps. The time step was $\Delta t=0.001 \sigma \sqrt{M / \varepsilon}$. The statistical uncertainty of the Henry's law constant is estimated to be three times the standard deviation of ten block averages, each sampling 100,000 time steps.

\subsection{Density gradient theory and equation of state}

\section{Density gradient theory}

Density gradient theory is applied here to binary systems with a planar interface, such that there is only a dependence on one spatial coordinate, which is labelled $z$ here. The free energy $a$ is expanded in a Taylor series in derivatives of the densities of the components with respect to the spatial coordinate $z\left(\nabla^{m} \boldsymbol{\rho} ; m=1,2, ..\right)$ and truncated after the square gradient term [64]:

$$
a[\boldsymbol{\rho}]=a_{0}(\boldsymbol{\rho})+\sum_{i=1}^{2} \sum_{j=1}^{2} \frac{1}{2} \kappa_{i j} \nabla \rho_{i} \cdot \nabla \rho_{j}
$$

where $a_{0}$ is the free energy of the homogeneous mixture at the local density and composition and $\boldsymbol{\rho}$ indicates the vector of the number densities of the components and $\nabla \rho$ the vector of the corresponding gradients. $\kappa_{i j}$ are the influence parameters, for the pure substances (if $i=j$ ) and the cross interaction (if $i \neq j$ ). The cross interaction influence parameter was computed as the geometric mean of the pure substance influence parameters $[41,65,66]$, i.e. $\kappa_{i j}=\sqrt{\kappa_{i i} \kappa_{j j}}$. The stabilized DGT (sDGT) algorithm proposed by Mu et al. [67] was applied for solving Eq. (7) and the computation of the surface tension. The domain length was in all cases set to $20 \sigma$ with a spatial discretisation of $0.02 \sigma$.

\section{EOS for the LJ and LJTS fluid}

The LJ and LJTS EOS from earlier works of group [51, 52] were employed for computing phase equilibria and interfacial properties with DGT. The DGT influence parameters $\kappa_{i i}$ for the LJ EOS and the LJTS EOS were parametrised to surface tension data from molecular simulation [51, 52].

The VLE computed from the LJ and LJTS EOS [51, 52] are compared in Fig. 2 with corresponding computer experiment data from the literature. Both EOS describe the VLE of the pure components very well. Details on the performance of both EOS can be found in the original publications [51, 52]. Also, the critical points from both EOS are in good agreement with those obtained from molecular simulations, cf. Table 1.

The long-range interactions applied for the LJ fluid significantly increase the critical temperature and pressure compared to the LJTS fluid but hardly affect the critical density [12-15]. Furthermore, 
the critical parameters are influenced more strongly by the long-range interactions than the triple point parameters. The vapour pressure and the enthalpy of vaporization are influenced more strongly by the long-range interactions than the saturated densities.

\subsection{Definition of interfacial properties derived from density profiles}

The direct VLE MD simulations as well as the DGT calculations yield density profiles of both components $\rho_{1}(z)$ and $\rho_{2}(z)$ at the planar interface. The relative adsorption and enrichment of the low-boiling component and the interfacial thickness were computed from these density profiles.

The relative adsorption $\Gamma_{2}^{(1)}$ of the low-boiling component 2 at the interface was computed by the symmetric interface segregation according to Telo da Gama and Evans [39] by

$$
\Gamma_{2}^{(1)}=-\left(\rho_{2}^{\prime}-\rho_{2}^{\prime \prime}\right) \int_{-\infty}^{\infty}\left[\frac{\rho_{1}(z)-\rho_{1}^{\prime}}{\rho_{1}^{\prime}-\rho_{1}^{\prime \prime}}-\frac{\rho_{2}(z)-\rho_{2}^{\prime}}{\rho_{2}^{\prime}-\rho_{2}^{\prime \prime}}\right] \mathrm{d} z
$$

where $\rho_{1}^{\prime}, \rho_{1}^{\prime \prime}$ and $\rho_{2}^{\prime}, \rho_{2}^{\prime \prime}$ are the saturated densities in the bulk liquid and bulk vapor phase, respectively.

The non-monotonicity of the density profiles is quantified by the enrichment as proposed by Becker et al. [42]. The enrichment of the low-boiling component $E_{2}$ is defined as the ratio of the maximum local density of the low-boiling component in the interfacial region and the larger of the corresponding component densities in the two bulk phases [41]:

$$
E_{2}=\frac{\max \left(\rho_{2}(z)\right)}{\max \left(\rho_{2}^{\prime}, \rho_{2}^{\prime \prime}\right)}
$$

The interfacial thickness $L_{10}^{90}$ is defined according to Lekner and Henderson [68] and measures the distance between the points where the total density $\rho_{\text {tot }}(z)=\rho_{1}(z)+\rho_{2}(z)$ reaches $10 \%$ and $90 \%$ of the total bulk densities respectively:

$$
\begin{aligned}
& L_{10}^{90}=z\left(\rho_{90}^{\mathrm{tot}}\right)-z\left(\rho_{10}^{\mathrm{tot}}\right), \\
& \rho_{10}^{\mathrm{tot}}=\rho_{\mathrm{tot}}^{\prime \prime}+0.1\left(\rho_{\mathrm{tot}}^{\prime}-\rho_{\mathrm{tot}}^{\prime \prime}\right), \\
& \rho_{90}^{\mathrm{tot}}=\rho_{\mathrm{tot}}^{\prime \prime}+0.9\left(\rho_{\mathrm{tot}}^{\prime}-\rho_{\mathrm{tot}}^{\prime \prime}\right) .
\end{aligned}
$$

\section{Results and discussion}

First, the interfacial properties obtained for pure fluids described by the LJ potential and LJTS potential are compared. Subsequently, the results for the phase equilibria and interfacial properties of the mixtures obtained from both potentials are compared. The numerical values of the results for the 
phase equilibria and the interfacial properties obtained in this work are presented in the Supplementary Material.

\subsection{Interfacial properties of pure substances}

Results from the present work for the surface tension and the interfacial thickness of the pure LJ fluid and the pure LJTS fluid are compared in Fig. 3. Besides the results from molecular simulation, also results fro EOS+DGT are shown, that were obtained after an adjustment of a state-independent number for $\kappa$ to simulation data of the surface tension as described in Refs. $[52,56]$ resulting in $\kappa_{\mathrm{LJ}}=5.10911$, and $\kappa_{\mathrm{LJTS}}=2.7334$.

The surface tension of both components decreases monotonically from the triple point temperature to the critical temperature. Starting at the triple point temperature, the interfacial thickness increases only slightly with increasing temperature but exhibits a strong convex trend and a pole at the critical temperature.

For both the LJ potential and the LJTS potential, computer experiment data for the surface tension from the literature are shown for comparison. In a recent study [53], surface tension data of the LJ fluid was reviewed and assessed. The results of Mecke et al. [32] and Werth et al. [18] were found to be precise and accurate [53] and are used as reference here. The LJ surface tension results from the present work are in excellent agreement with those of Refs. [18, 32]. The LJTS surface tension results from the present work are in good agreement with those reported by Vrabec et al. [69], who found their data to be in excellent agreement with other reliable literature data.

At the same reduced temperature, the LJ fluid has a larger surface tension and interfacial thickness than the LJTS fluid. The long-range interactions have no significant influence on the shape of $\gamma(T)$ and $L_{10}^{90}(T)$, except the shifted critical point. This is in line with the results reported by Dunikov et al. [13] and Baidakov et al. [21]. The long-range interactions have a stronger influence on the surface tension than on the interfacial thickness - especially at lower temperatures. At the same reduced temperature, the surface tension of the LJ fluid is up to about $40 \%$ higher than that of the LJTS fluid, indicating a strong influence of the long-range interactions on this property. Evidently, these differences vanish at the critical point $T / T_{\mathrm{c}, 1}=1$. Also the interfacial thickness of the LJ fluid is larger than that of the LJTS fluid, but only by about $15 \%$. Also this increase is expected as a consequence of the long-range interactions[13].

For both the LJ potential and the LJTS potential, the EOS+DGT results are in good agreement with the results from the corresponding computer experiments. The agreement is better for the surface tension than it is for the interfacial thickness, as $\gamma$ was used for the fitting of the DGT influence parameter $[52,56]$, while the EOS+DGT results for the interfacial thickness are predictions. For $L_{10}^{90}$, 
the agreement of computer experiment and EOS+DGT is slightly better for the LJ fluid compared to the LJTS fluid. The deviations between the MD results and those from EOS+DGT are likely due to fluctuations at the interface which are present in MD but not in DGT.

\subsection{Phase equilibria of binary mixtures}

Fig. 4 shows the results for the Henrys law constants $H_{2,1}$ of the supercritical component 2 in the solvent 1 for the mixtures D - F. Results for the LJ potential and LJTS potential are compared. Both MD simulation results and results from the corresponding EOS are shown. Results for the entire temperature range between the triple point and the critical point of the solvent 1 are shown. The results for the three systems differ strongly. This is a consequence of the differences in the binary interaction parameter $\xi_{12}$, that controls the strength of the unlike attractive interactions $1-2$, which are highly important for the gas solubility: the stronger the attraction 1 - 2 (the higher $\xi_{12}$ ), the better the solubility (the lower $H_{2,1}$ ). The variation of $\xi_{12}$ between 0.85 (mixture F) and 1.2 (mixture E) leads to differences in $H_{2,1}$ of almost an order of magnitude. Not only the numbers of $H_{2,1}$ are strongly affected by $\xi_{12}$, but also the temperature dependence $H_{2,1}(T)$, which is related to the enthalpy of absorption of the solute 2 in the solvent 1 . Strong unlike attractive interactions lead to exothermal absorption and a positive slope on $H_{2,1}(T)$ (mixture E), weak unlike attractive interactions lead to endothermal absorption and a negative slope of $H_{2,1}(T)$ (mixture F). In the ideal mixture, a maximum of $H_{2,1}(T)$ is found. All these observations hold both for the results from the LJ potential and those from the LJTS potential.

Figure 4 gives also insights on the influence of the long-range interactions on the Henrys law constants $H_{2,1}$. This influence depends strongly on $\xi_{12}$. For strong unlike attractive interactions $\left(\xi_{12}=1.2\right.$, mixture E) neglecting the long-range part leads to a decrease of the solubility (increase of $H_{2,1}$ ), as might be expected. The inverse trend is observed for mixture F. This is astonishing, but can be understood by the fact that not only the solute-solvent interactions 1 - 2 are subject to the truncation but also the solvent-solvent interactions 1 - 1 . Neglecting the long-range interactions 1 - 1 facilitates the insertion of 2 and increases the solubility (decreases $H_{2,1}$ ). Both effects compete in the ideal mixture D.

The results from the EOS and those from the computer experiments agree overall well. The best agreement is observed for mixture $\mathrm{D}$, while for the mixtures $\mathrm{E}$ and $\mathrm{F}$ deviations up to $10 \%$ are found. The EOS underestimate the results from the computer experiment for mixture E but overestimate them for mixture F. These findings hold for both the results of the LJ fluid and the LJTS fluid, which may be related to the fact that both EOS have the same functional form [51, 52].

Fig. 5, panels A - F, show the binary phase diagrams obtained from the LJ potential and LJTS 
potential at the same reduced temperature and pressure. The influence of the interaction parameters $\varepsilon_{2} / \varepsilon_{1}$ and $\xi_{12}$ on the phase behaviour of Lennard-Jones mixtures has already been discussed elsewhere [41].

Fig. 5 reveals that the long-range interactions have practically no influence on the type of phase behaviour of mixtures. As the weakening of the attractive interactions by cutting off the long-range interactions affects all interactions that occur in the mixture (those between the same partners as well as those between unlike partners), the qualitative phase behaviour is not influenced. E.g., the position of the azeotropic points in the systems B and C is almost the same for the LJ potential and LJTS potential. As for the Henry's law constants, the results from the EOS and the computer experiments are in good agreement. This holds for both the LJ and the LJTS potential.

For the mixture A and B, the results obtained for the LJ potential and LJTS potential agree almost perfectly when reduced variables are used, as in Fig. 5. For the mixtures $\mathrm{C}$ - F, the MD results obtained for the LJ potential and LJTS potential show small deviations - mainly for the bubble line. The EOS results for the mixtures D - F for the LJ potential and LJTS potential show distinct deviations close to the critical point, which is mainly due to the fact that all results were reduced with the computer experiment critical pressure and temperature of the respective potential.

Fig. 6, panels A - F, show the binary saturated density phase diagrams obtained from the LJ potential and LJTS potential at $T / T_{\mathrm{c}, 1}=0.7$. The saturated liquid density $\rho^{\prime}$ and the saturated vapour density $\rho^{\prime \prime}$ are shown as a function of the pressure. The results obtained from the LJ and the LJTS potential show only little differences, as expected from the pure substance properties, cf. Fig. 2. Hence, the long range interactions have no significant influence on the saturated densities of the mixtures. The results obtained from both the LJ and LJTS EOS agree very well with the corresponding computer experiments.

For the mixture A (ideal mixture), the saturated densities show a monotonous and almost linear transition between the two pure component density values. For the mixture B and C on the other hand, the saturated densities show a more complex behaviour. For the mixture B (high-boiling azeotrope), starting at the pure component 1 , the difference of the two densities $\Delta \rho=\rho^{\prime}-\rho^{\prime \prime}$ first increases with increasing pressure until the azeotropic point and then decreases with further increasing pressure until the pure component 2 value is reached; vice versa for the mixture C. Hence, the difference of the two densities $\Delta \rho=\rho^{\prime}-\rho^{\prime \prime}$ exhibits a minimum as a function of the pressure in the mixture $\mathrm{C}$ and a maximum in the mixture $\mathrm{B}$. 


\subsection{Interfacial properties of binary mixtures}

The influence of the mixture type A - F on vapour-liquid interfacial properties has been studied before [41]. The following discussion therefore focuses exclusively on the influence of the long-range interactions on the interfacial properties of the binary mixtures.

Fig. 7, panels A - F, show the results for the surface tension obtained from the LJ potential and LJTS potential. The EOS+DGT and the simulation results agree almost always within the MD error bars. Slight deviations are found for the mixtures B, E, and F. Overall, the EOS+DGT results describe the results of the computer experiment for the surface tension similarly well for both the for the LJ potential and LJTS potential.

As for the pure substance surface tension, the long-range interactions have an important influence on the surface tension of binary mixtures. The long-range interactions present in the LJ simulations increase the surface tension for all six investigated mixtures compared to the LJTS results. The surface tension obtained from the LJ potential exceeds that from the LJTS potential by $40-50 \%$. Nevertheless, the shape of $\gamma\left(x_{2}^{\prime}\right)$ is hardly affected by the long-range interactions. Also the aneotropic composition (minimum and maximum of $\gamma\left(x_{2}^{\prime}\right)$ ) [70-72] is only slightly affected by the long-range interactions.

Fig. 8, panels A - F, show the results for the interfacial thickness obtained from the LJ potential and LJTS potential. As for the pure substances (cf. Fig. 3), the interfacial thickness of the LJ mixtures exceed those of the LJTS mixtures. The average difference of the LJ and LJTS computer experiment results for the interfacial thickness is approximately $10 \%$. As for the surface tension, these differences between the LJ and the LJTS fluid are practically composition-independent. The shape of $L_{10}^{90}\left(x_{2}^{\prime}\right)$ for the LJ potential and LJTS potential are similar.

The $L_{10}^{90}\left(x_{2}^{\prime}\right)$ results from MD and EOS+DGT agree very well for the case of the LJ potential. In contrast, for the results of the LJTS potential, the MD simulations are generally underestimated by the EOS+DGT. This indicates a weakness of the the EOS that was used for describing the LJTS fluid. A major difference of that EOS as compared to the one used for describing the LJ fluid is that in the parametrisation of the latter, also homogeneous bulk properties were used [41]. On the other hand, the employed LJTS EOS was exclusively parametrised to VLE data [52].

Fig. 9, panels A - F, show the results for the relative adsorption $\Gamma_{2}^{(1)}$ of the low-boiling component at the vapour-liquid interface (cf. Eq. (8)). The long-range interactions only have a minor influence on the relative adsorption of the investigated Lennard-Jones mixtures. This is due to the fact that the relative adsorption $\Gamma_{2}^{(1)}$ is closely linked to the slope of the surface tension $\gamma\left(x_{2}^{\prime}\right)$ via the Gibbs adsorption equation and the composition dependency of $\gamma\left(x_{2}^{\prime}\right)$ is hardy affected by the long-range interactions, cf. Fig. 7. The MD results for $\Gamma_{2}^{(1)}$ of the mixtures A - F obtained from the LJ potential and LJTS potential agree within their statistical uncertainties. Nevertheless, $\Gamma_{2}^{(1)}$ from the LJ potential 
is in most cases slightly higher than the corresponding LJTS results. The EOS+DGT results support this tendency.

For the mixtures A \& D - F, the relative adsorption is always positive $\Gamma_{2}^{(1)}>0$, i.e. the light boiling component adsorbs at the interface in the entire investigate composition range. For the mixtures B \& $\mathrm{C}$, the relative adsorption exhibits a range of positive and a range of negative adsorption connected by a zero crossing $\Gamma_{2}^{(1)}=0$, which is a result of the aneotropic behaviour. For all mixtures and compositions where $\Gamma_{2}^{(1)}>0$, the relative adsorption obtained by the LJ potential slightly exceeds that obtained by the LJTS potential: $\Gamma_{2, \mathrm{LJ}}^{(1)}>\Gamma_{2, \mathrm{LJTS}}^{(1)}$. For the composition range of the mixture B \& $\mathrm{C}$ where $\Gamma_{2}^{(1)}<0$, results obtained for the LJ potential yield a stronger desorption of the light boiling component at the interface than the corresponding results for the LJTS potential.

The long-range interactions have no significant influence on the slope of $\Gamma_{2}^{(1)}\left(x_{2}^{\prime}\right)$.

As for the surface tension results (cf. Fig. 7), the EOS+DGT results of $\Gamma_{2}^{(1)}\left(x_{2}^{\prime}\right)$ agree well with the corresponding MD results.

Fig. 10, panels $\mathrm{A}-\mathrm{F}$, show the results for the enrichment $E_{2}$ of the low-boiling component at the vapour-liquid interface (cf. Eq. (9)) in the six studied mixtures. Large enrichment is only found in the mixtures $\mathrm{D}$ and $\mathrm{F}$. The influence of the long-range interactions on the enrichment of the low-boiling component at the interface is marginal. The results from the computer experiments for $E_{2}$ obtained from the LJ potential and LJTS potential agree within their statistical uncertainties for most mixtures. Nonetheless, there is a trend that the results obtained from the LJ potential are slightly higher than those obtained from the LJTS potential. This is not unexpected as the enrichment is important at low $x_{2}^{\prime}$ where the interactions 1 - 2 are dominant, which are weaker when the potential is truncated.

While the results from EOS+DGT agree very well with the computer experiment for the other interfacial properties discussed above, this does not hold for the enrichment $E_{2}$. The results agree only qualitatively. As already discussed in Refs. [40-42], partially significant differences between results from EOS+DGT and computer experiments are observed. For the EOS+DGT, the LJ and LJTS results show the same trends but partially significant deviations from the MD data are observed. Furthermore, there are also significant differences between the EOS+DGT results obtained for the LJ potential and the LJTS potential in some mixtures, whereas such differences are not found in the MD results. As discussed above, the differences in the qualities of the EOS might play a role also here. The agreement for the enrichment obtained by EOS+DGT and computer experiments is slightly better for the LJ potential than the LJTS potential (cf. Fig. 10 - C and D). The enrichment is the most sensitive of the investigated properties - regarding the agreement of the theory and computer experiments - in this study. 


\section{Conclusions}

In the present work, the influence of long-range interactions on thermophysical properties of mixtures was systematically investigated for the first time. For that purpose, six binary Lennard-Jones mixtures were studied with the LJ potential and the LJTS potential. The comparison was carried out at the same reduced temperature of the heavy-boiling component 1. Different components 2 (subcritical and supercritical) were used. Also the strength of the unlike interactions $1-2$ was varied, such that six different types of phase behaviours were obtained.

The truncation and shifting of the potential in the LJTS fluid weakens the attractive interactions compared to the LJ fluid. This affects pure component properties in ways that are well-known from previous studies: e.g., the vapour pressure of the LJ fluid is lower than that of the LJTS fluid at the same temperature $[12,13,15,21,25]$. In binary mixtures, the reduced attractive interactions in the LJTS potential compared to the full LJ potential affects all interactions in the same way, the like ones as well as the unlike ones. Hence, it is expected that the qualitative behaviour of the LJ mixtures and the corresponding LJTS mixtures should be similar. This is confirmed in the present study for different mixture types and all bulk and interfacial properties that were investigated: the concentration-dependence that is observed for the different properties is always similar for the LJ and the LJTS mixtures. The sign of the offset between the results depends on the studied property and can generally be explained by the weaker attractive interactions in the LJTS fluid. As for the pure fluids, the surface tension in mixtures that is obtained from the LJ potential is always higher than the corresponding number obtained from the LJTS potential, whereas the interfacial thickness is only slightly increased. In contrast to the surface tension $\gamma$, the relative adsorption $\Gamma_{2}^{(1)}$, which is related to the slope of $\gamma\left(x_{2}^{\prime}\right)$, is almost the same for the LJ fluid and the LJTS fluid. Furthermore, no significant influence of the long-range interactions on the enrichment at interfaces was observed.

Henry's law constants obtained for LJ and LJTS mixtures differ in general. The differences may have different signs, depending on the strength of the unlike interactions, that was varied here using the parameter $\xi_{12}$.

All studies of the present work were carried out not only with MD simulations but also using EOS for the LJ and the LJTS fluid - for the interfacial properties in connection with DGT. The EOS and EOS+DGT calculations confirm the findings from the MD simulations.

\section{Acknowledgements}

The authors gratefully acknowledge funding of the present work by the ERC Advanced Grant ENRICO (grant agreement No. 694807). The simulations were carried out on the ELWE supercomputer at 
Regional University Computing Center Kaiserslautern (RHRK) under the grant TUK-TLMV. The present research was conducted under the auspices of the Boltzmann-Zuse Society of Computational Molecular Engineering (BZS).

\section{Disclosure statement}

No potential conflict of interest was reported by the authors.

\section{Funding}

ERC Advanced Grant ENRICO [grant agreement No. 694807]. 


\section{References}

[1] Allen MP, Tildesley DJ. Computer simulation of liquids. Oxford: Oxford University Press; 1989.

[2] Martin MG, Siepmann JI. Transferable potentials for phase equilibria. 1. United-atom description of nalkanes. J Phys Chem B. 1998;102(14):2569-2577. Available from: https://doi .org/10 .1021/jp972543+.

[3] Stephan S, Horsch M, Vrabec J, Hasse H. MolMod - an open access database of force fields for molecular simulations of fluids. Mol Simulat. 2019;45(10):806-814.

[4] Nicolas JJ, Gubbins KE, Streett WB, Tildesley DJ. Equation of state for the Lennard-Jones fluid. Molecular Physics. 1979;37(5):1429.

[5] Powles J, Evans W, Quirke N. Non-destructive molecular-dynamics simulation of the chemical potential of a fluid. Mol Phys. 1982;46(6):1347-1370. Available from: https://doi.org/10.1080/00268978200101981.

[6] Janeček J. Long range corrections in inhomogeneous simulations. The Journal of Physical Chemistry B. 2006;110(12):6264-6269.

[7] Werth S, Rutkai G, Vrabec J, Horsch M, Hasse H. Long-range correction for multi-site Lennard-Jones models and planar interfaces. Molecular Physics. 2014;112(17):2227-2234.

[8] Lishchuk SV, Fischer J. Communication: Long range corrections in liquid-vapor interface simulations. J Chem Phys. 2018;149(9):091102.

[9] Meja A, Vega LF. Perfect wetting along a three-phase line: Theory and molecular dynamics simulations. The Journal of Chemical Physics. 2006;124(24):244505.

[10] Müller EA, Mejía A. Interfacial properties of selected binary mixtures containing n-alkanes. Fluid Phase Equilibria. 2009;282(2):68-81.

[11] Lobanova O, Mejía A, Jackson G, Müller EA. SAFT-i force field for the simulation of molecular fluids 6: Binary and ternary mixtures comprising water, carbon dioxide, and n-alkanes. The Journal of Chemical Thermodynamics. 2016;93:320-336.

[12] Shi W, Johnson J. Histogram reweighting and finite-size scaling study of the Lennard-Jones fluids. Fluid Phase Equilibria. 2001;187:171-191.

[13] Dunikov DO, Malyshenko SP, Zhakhovskii VV. Corresponding states law and molecular dynamics simulations of the Lennard-Jones fluid. J Chem Phys. 2001;115(14):6623-6631.

[14] Powles J. The liquid-vapour coexistence line for Lennard-Jones-type fluids. Physica A: Statistical Mechanics and its Applications. 1984;126(1):289-299. Available from: http://www.sciencedirect.com/science/ article/pii/0378437184901560.

[15] Smit B. Phase diagrams of Lennard-Jones fluids. J Chem Phys. 1992;96(11):8639-8640. Available from: https://doi.org/10.1063/1.462271.

[16] Lotfi A, Vrabec J, Fischer J. Vapour liquid equilibria of the Lennard-Jones fluid from the NpT plus test particle method. Mol Phys. 1992;76(6):1319-1333.

[17] Trokhymchuk A, Alejandre J. Computer simulations of liquid/vapor interface in Lennard-Jones fluids: Some questions and answers. J Chem Phys. 1999;111(18):8510-8523. 
[18] Werth S, Lishchuk SV, Horsch M, Hasse H. The influence of the liquid slab thickness on the planar vaporliquid interfacial tension. Physica A. 2013;392(10):2359.

[19] Guo M, Peng DY, C-YLu B. On the long-range corrections to computer simulation results for the LennardJones vapor-liquid interface. Fluid Phase Equilibr. 1997;130(1):19-30.

[20] Nijmeijer MJP, Bakker AF, Bruin C, Sikkenk JH. A molecular dynamics simulation of the Lennard-Jones liquid-vapor interface. J Chem Phys. 1988;89(6):3789-3792.

[21] Baidakov V, Chernykh G, Protsenko S. Effect of the cut-off radius of the intermolecular potential on phase equilibrium and surface tension in Lennard-Jones systems. Chem Phys Lett. 2000;321(3):315-320.

[22] Evans R, Henderson JR, Hoyle DC, Parry AO, Sabeur ZA. Asymptotic decay of liquid structure: Oscillatory liquid-vapour density profiles and the Fisher-Widom line. Molecular Physics. 1993;80(4):755-775.

[23] Evans R. Oscillatory behaviour of density profiles: Relevance for fluid interfacial phenomena. Berichte der Bunsengesellschaft für physikalische Chemie. 1994;98(3):345-352.

[24] Johnson JK, Zollweg JA, Gubbins KE. The Lennard-Jones equation of state revisited. Mol Phys. 1993; $78(3): 591$.

[25] Shaul KRS, Schultz AJ, Kofke DA. The effect of truncation and shift on virial coefficients of Lennard-Jones potentials. Collect Czech Chem C. 2010;75(4):447-462.

[26] Blokhuis E, Bedeaux D, Holcomb C, Zollweg J. Tail corrections to the surface tension of a Lennard-Jones liquid-vapour interface. Molecular Physics. 1995;85(3):665-669.

[27] Shen VK, Mountain RD, Errington JR. Comparative study of the effect of tail corrections on surface tension determined by molecular simulation. J Phys Chem B. 2007;111(22):6198-6207.

[28] Janeček J, Said-Aizpuru O, Paricaud P. Long range corrections for inhomogeneous simulations of Mie n-m potential. J Chem Theory Comput. 2017;13(9):4482-4491.

[29] Guo M, Lu BCY. Long range corrections to thermodynamic properties of inhomogeneous systems with planar interfaces. J Chem Phys. 1997;106(9):3688-3695.

[30] Holcomb CD, Clancy P, Zollweg JA. A critical study of the simulation of the liquid-vapour interface of a Lennard-Jones fluid. Mol Phys. 1993;78(2):437-459.

[31] Martinez-Ruiz FJ, Blas FJ, Mendiboure B, Moreno-Ventas Bravo AI. Effect of dispersive long-range corrections to the pressure tensor: The vapour-liquid interfacial properties of the Lennard-Jones system revisited. J Chem Phys. 2014;141(18):184701.

[32] Mecke M, Winkelmann J, Fischer J. Molecular dynamics simulation of the liquid-vapor interface: The Lennard-Jones fluid. J Chem Phys. 1997;107(21):9264-9270.

[33] Lu BQ, Evans R, Telo da Gama MM. The form of the density profile at a liquid-gas interface. Molecular Physics. 1985;55(6):1319-1338.

[34] Ibergay C, Ghoufi A, Goujon F, Ungerer P, Boutin A, Rousseau B, Malfreyt P. Molecular simulations of the $n$-alkane liquid-vapor interface: Interfacial properties and their long range corrections. Physical Review E. 2007;75:051602.

[35] Grosfils P, Lutsko JF. Dependence of the liquid-vapor surface tension on the range of interaction: A test 
of the law of corresponding states. The Journal of Chemical Physics. 2009;130(5):054703.

[36] Ghoufi A, Malfreyt P, Tildesley DJ. Computer modelling of the surface tension of the gas-liquid and liquid-liquid interface. Chem Soc Rev. 2016;45:1387-1409.

[37] in 't Veld PJ, Ismail AE, Grest GS. Application of Ewald summations to long-range dispersion forces. The Journal of Chemical Physics. 2007;127(14):144711.

[38] Lee DJ, Telo da Gama MM, Gubbins KE. The vapour-liquid interface for a Lennard-Jones model of argonkrypton mixtures. Molecular Physics. 1984;53(5):1113-1130.

[39] Telo da Gama MM, Evans R. The structure and surface tension of the liquid-vapour interface near the upper critical end point of a binary mixture of Lennard-Jones fluids. Molecular Physics. 1983;48(2):229-250.

[40] Stephan S, Langenbach K, Hasse H. Enrichment of components at vapour-liquid interfaces: A study by molecular simulation and density gradient theory. Chemical Engineering Transactions. 2018;69:295-300.

[41] Stephan S, Langenbach K, Hasse H. Interfacial properties of binary Lennard-Jones mixtures by molecular simulations and density gradient theory. The Journal of Chemical Physics. 2019;150(17):174704.

[42] Becker S, Werth S, Horsch M, Langenbach K, Hasse H. Interfacial tension and adsorption in the binary system ethanol and carbon dioxide: Experiments, molecular simulation and density gradient theory. Fluid Phase Equilibria. 2016;427:476.

[43] Klink C, Gross J. A density functional theory for vapor-liquid interfaces of mixtures using the perturbedchain polar statistical associating fluid theory equation of state. Industrial \& Engineering Chemistry Research. 2014;53(14):6169.

[44] Telo da Gama M, Evans R. The structure and surface tension of the liquid-vapour interface near the upper critical end point of a binary mixture of Lennard-Jones fluids. Molecular Physics. 1983;48(2):251-266.

[45] Lee DJ, Telo da Gama MM, Gubbins KE. Adsorption and surface tension reduction at the vapor-liquid interface. The Journal of Physical Chemistry. 1985;89(8):1514-1519.

[46] Protsenko SP, Baidakov VG, Bryukhanov VM. Binary Lennard-Jones mixtures with highly asymmetric interactions of the components. 2. effect of the particle size on phase equilibria and properties of liquid-gas interfaces. Fluid Phase Equilibr. 2016;430:67-74.

[47] Protsenko SP, Baidakov VG. Binary Lennard-Jones mixtures with highly asymmetric interactions of the components. 1. effect of the energy parameters on phase equilibria and properties of liquid-gas interfaces. Fluid Phase Equilibr. 2016;429:242-253.

[48] Mejía A, Pàmies JC, Duque D, Segura H, Vega LF. Phase and interface behaviors in type-I and type-V Lennard-Jones mixtures: Theory and simulations. The Journal of Chemical Physics. 2005;123(3):034505.

[49] Garrido JM, Quinteros-Lama H, Pieiro MM, Mejía A, Segura H. On the phase and interface behavior along the three-phase line of ternary Lennard-Jones mixtures: A collaborative approach based on square gradient theory and molecular dynamics simulations. The Journal of Chemical Physics. 2014;141(1):014503.

[50] Buhn JB, Bopp PA, Hampe MJ. A molecular dynamics study of a liquid-liquid interface: Structure and dynamics. Fluid Phase Equilibria. 2004;224(2):221-230.

[51] Stephan S, Staubach J, Forte E, Hasse H. Equations of state of the Lennard-Jones fluid: a systematic 
comparison. in preparation. 2019;

[52] Heier M, Stephan S, Liu J, Chapman WG, Hasse H, Langenbach K. Equation of state for the Lennard-Jones truncated and shifted fluid with a cut-off radius of $2.5 \sigma$ based on perturbation theory and its applications to interfacial thermodynamics. Molecular Physics. 2018;116(15):2083-2094.

[53] Stephan S, Thol M, Vrabec J, Hasse H. Thermophysical properties of the lennard-jones fluid: Database and data assessment. Journal of Chemical Information and Modeling. 2019;59(10):4248-4265.

[54] Lorentz HA. Ueber die Anwendung des Satzes vom Virial in der kinetischen Theorie der Gase. Annalen der Physik. 1881;248:127-136.

[55] Berthelot D. Sur le mélange des gaz. Comptes rendus hebdomadaires des séances de l'Académie des Sciences. 1898;126:1703-1706.

[56] Stephan S, Liu J, Langenbach K, Chapman WG, Hasse H. Vapor-liquid interface of the Lennard-Jones truncated and shifted fluid: Comparison of molecular simulation, density gradient theory, and density functional theory. J Phys Chem C. 2018;122(43):24705-24715.

[57] Thol M, Rutkai G, Span R, Vrabec J, Lustig R. Equation of state for the Lennard-Jones truncated and shifted model fluid. International Journal of Thermophysics. 2015;36(1):25.

[58] Niethammer C, Becker S, Bernreuther M, Buchholz M, Eckhardt W, Heinecke A, Werth S, Bungartz HJ, Glass CW, Hasse H, Vrabec J, Horsch M. ls1 mardyn: The massively parallel molecular dynamics code for large systems. Journal of Chemical Theory Computation. 2014;10(10):4455.

[59] Walton J, Tildesley DJ, Rowlinson JS, Henderson JR. The pressure tensor at the planar surface of a liquid. Molecular Physics. 1983;48(6):1357-1368.

[60] Kirkwood JG, Buff FP. The statistical mechanical theory of surface tension. The Journal of Chemical Physics. 1949;17(3):338-343.

[61] Rutkai G, Köster A, Guevara-Carrion G, Janzen T, Schappals M, Glass CW, Bernreuther M, Wafai A, Stephan S, Kohns M, Reiser S, Deublein S, Horsch M, Hasse H, Vrabec J. ms2: A molecular simulation tool for thermodynamic properties, release 3.0. Comput Phys Commun. 2017;221:343-351.

[62] Shing KS, Gubbins KE, Lucas K. Henry constants in non-ideal fluid mixtures. Molecular Physics. 1988; $65(5): 1235-1252$.

[63] Widom B. Some topics in the theory of fluids. J Chem Phys. 1963;39(11):2808.

[64] Rowlinson JS, Widom B. Molecular theory of capillarity. New York: Dover Publications; 1982.

[65] Carey BS, Scriven LE, Davis HT. Semiempirical theory of surface tension of binary systems. AIChE Journal. $1980 ; 26(5): 705-711$.

[66] Miqueu C, Mendiboure B, Graciaa C, Lachaise J. Modelling of the surface tension of binary and ternary mixtures with the gradient theory of fluid interfaces. Fluid Phase Equilibria. 2004;218(2):189-203.

[67] Mu X, Frank F, Alpak FO, Chapman WG. Stabilized density gradient theory algorithm for modeling interfacial properties of pure and mixed systems. Fluid Phase Equilibria. 2017;435:118-130.

[68] Lekner J, Henderson JR. Surface tension and energy of a classical liquid-vapour interface. Molecular Physics. $1977 ; 34(2): 333-359$. 
[69] Vrabec J, Kedia GK, Fuchs G, Hasse H. Comprehensive study of the vapour-liquid coexistence of the truncated and shifted Lennard-Jones fluid including planar and spherical interface properties. Molecular Physics. 2006;104(9):1509.

[70] McLure IA, Edmonds B, Lal M. Extremes in surface tension of fluorocarbon + hydrocarbon mixtures. Nature Physical Science. 1973;241(107):71-71.

[71] McLure IA, Soares VAM, Williamson AM. Total surface segregation. a fresh look at the Gibbs adsorption isotherm for binary liquid mixtures. Langmuir. 1993;9(8):2190-2201.

[72] Defay R, Prigogine I, Bellmans A, Everett DH. Surface tension and adsorption. London: Longmans; 1966. 
Table 1. Critical point properties (top) and triple point properties (bottom) of the LJ and LJTS fluid. The stated computer experiment (CE) values were estimated from a large collective of reported literature values[53, 56, 57]: The stated CE value is the mean average value and the corresponding uncertainty is estimated from their standard deviation. $\rho_{\mathrm{tr}}^{\prime}$ and $\rho_{\mathrm{tr}}^{\prime \prime}$ indicate the liquid and vapour density at the triple point.

\begin{tabular}{r|llll} 
& $T_{\mathrm{c}}$ & $\rho_{\mathrm{c}}$ & $p_{\mathrm{c}}$ \\
\hline LJ-CE & $1.321 \pm 0.007$ & $0.316 \pm 0.005$ & $0.129 \pm 0.005$ & \\
LJ-EOS [51] & 1.335 & 0.311 & 0.138 & \\
LJTS-CE & $1.078 \pm 0.017$ & $0.319 \pm 0.004$ & $0.098 \pm 0.007$ & \\
LJTS-EOS [52] & 1.089 & 0.309 & 0.102 & $p_{\mathrm{tr}}$ \\
\hline & $T_{\mathrm{tr}}$ & $\rho_{\mathrm{tr}}^{\prime}$ & $\rho_{\mathrm{tr}}^{\prime \prime}$ & $0.0015 \pm 0.0008$ \\
\hline LJ-CE & $0.66 \pm 0.024$ & $0.845 \pm 0.023$ & $0.019 \pm 0.0004$ & 0.0027
\end{tabular}




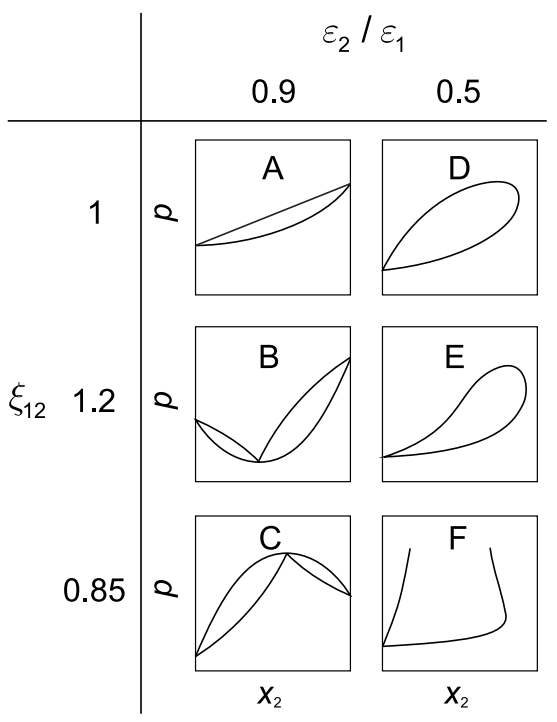

Figure 1. Sketches of the isothermal phase diagrams of the studied binary mixtures $1+2$. The different choices of the ratio of the dispersion energies $\varepsilon_{2} / \varepsilon_{1}$ and the binary interaction parameter $\xi_{12}$ lead to six different types of phase behaviour (A - F). 


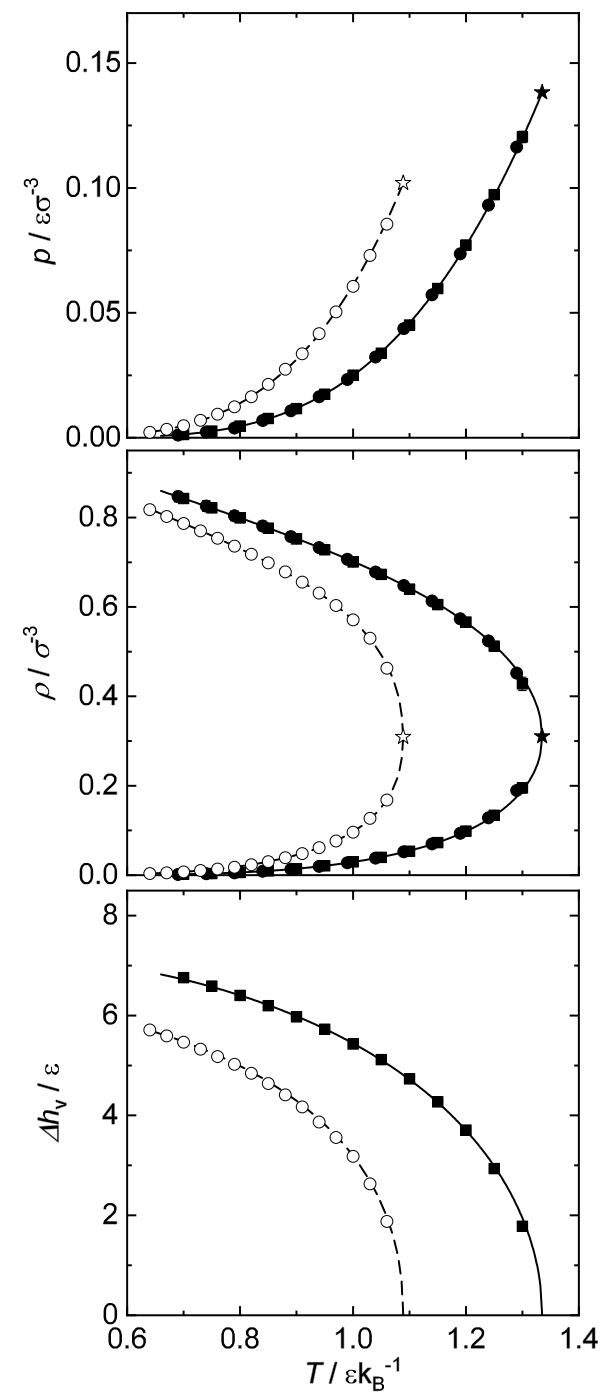

Figure 2. Vapour-liquid equilibrium of the LJ and LJTS fluid: vapour pressure (top), saturated densities (middle), enthalpy of vaporisation (bottom). Black lines and black symbols indicate the LJ fluid; dashed lines and open symbols indicate the LJTS fluid. Lines indicate the corresponding EOS [51,52], stars the critical point computed from the EOS. All other symbols are MD: • this work; - Lotfi et al. [16]; o Vrabec et al. [69]. 


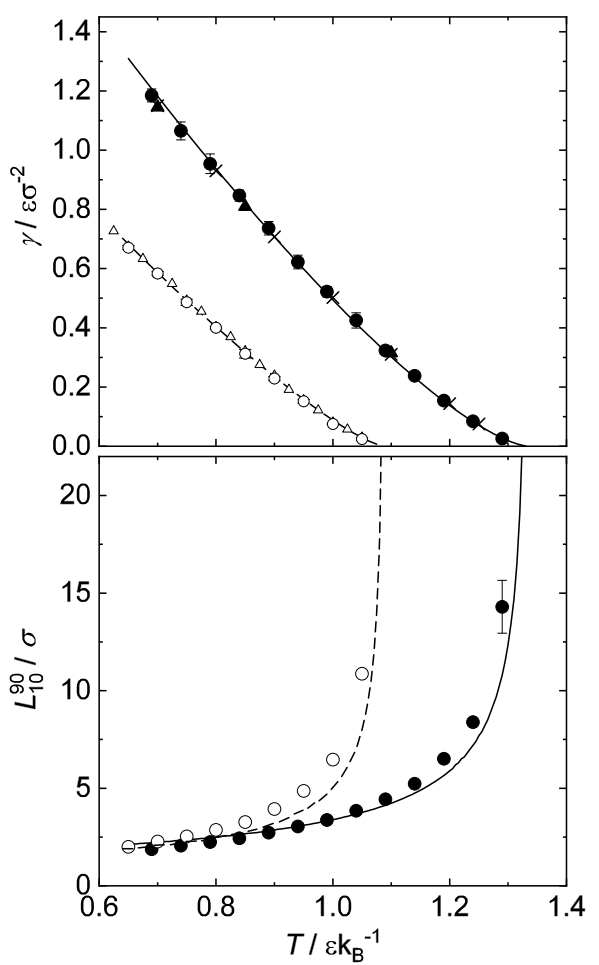

Figure 3. Vapour-liquid interfacial properties of the LJ and LJTS fluid: surface tension (top), interfacial thickness (bottom). Lines are EOS+DGT results: Solid lines indicate the LJ fluid; dashed lines indicate the LJTS fluid. Symbols are MD results. LJ fluid: • this work; $\Delta$ Mecke et al. [32]; $\times$ Werth et al. [18]. LJTS fluid: o this work; $\Delta$ Vrabec et al. [69].

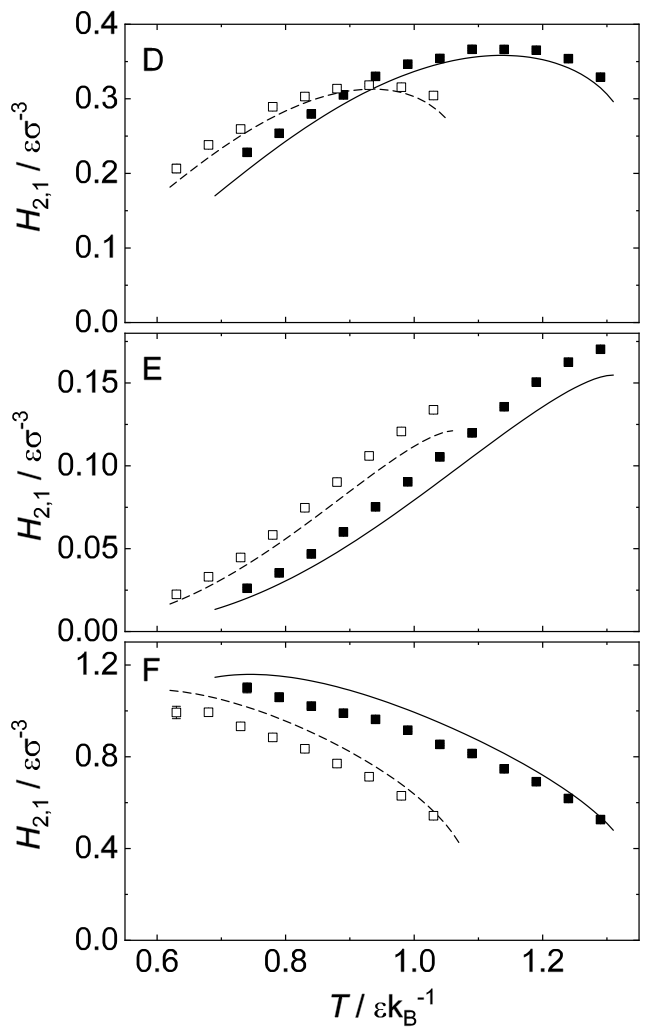

Figure 4. Henry's law constants of the binary mixtures D, E, and F between the triple and critical temperature. Symbols are MD results, lines are EOS results. Black lines and black symbols indicate the LJ potential; dashed lines and open symbols indicate the LJTS potential. 

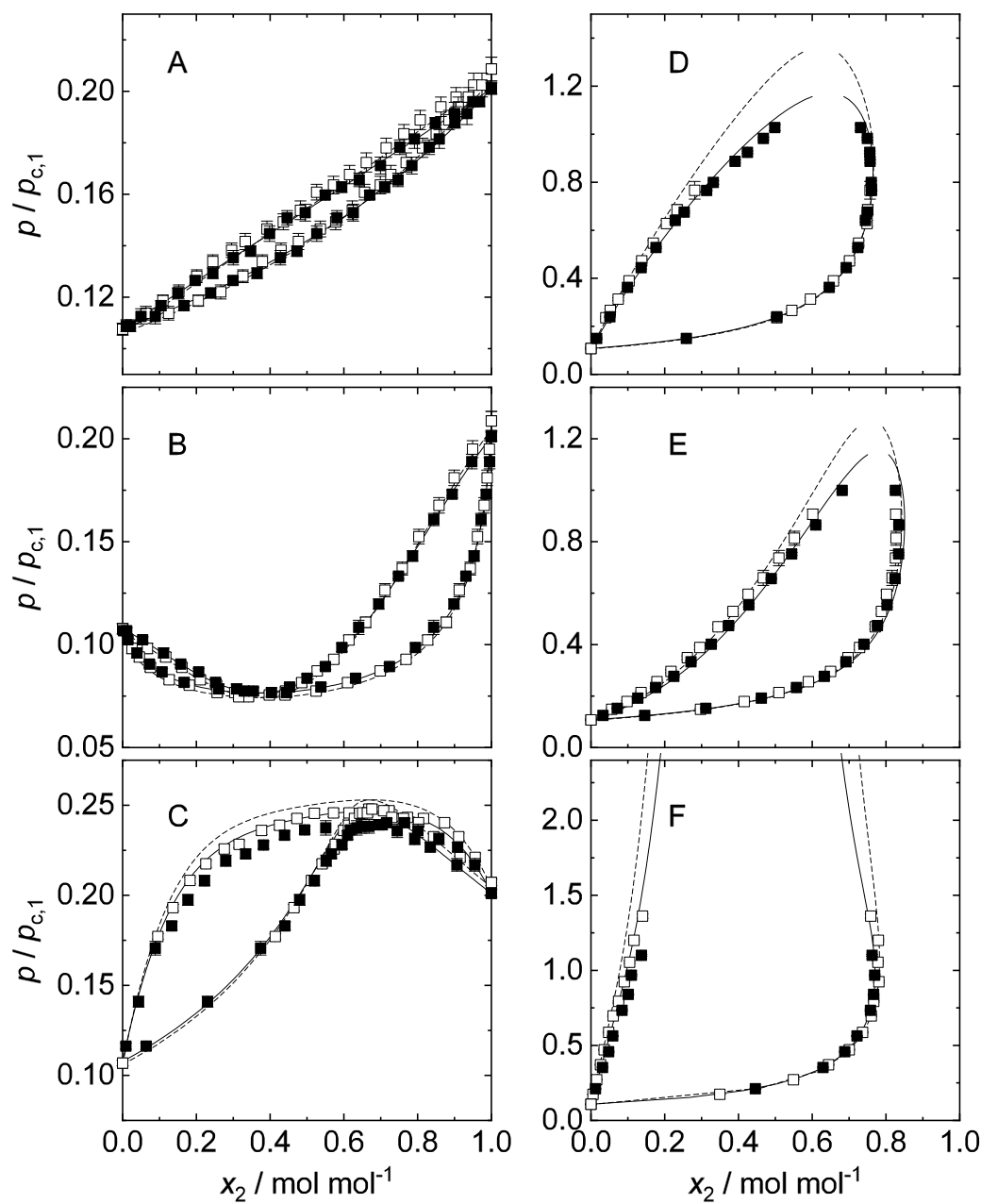

Figure 5. Vapour-liquid equilibria of the binary mixtures $\mathrm{A}, \mathrm{B}, \mathrm{C}, \mathrm{D}, \mathrm{E}$, and $\mathrm{F}$ at $T / T_{\mathrm{c}, 1}=0.7$, where $T_{\mathrm{c}, 1}$ is the critical temperature of the high-boiling component 1 . The pressure axis is reduced by critical pressure of the respective high-boiling component $\left(p_{\mathrm{c}, 1}=0.129\right.$ in the case of the LJ potential and $p_{\mathrm{c}, 1}=0.098$ in the case of the LJTS potential). Symbols are MD results, lines are EOS results. Black lines and black symbols indicate the LJ potential; dashed lines and open symbols indicate the LJTS potential. 

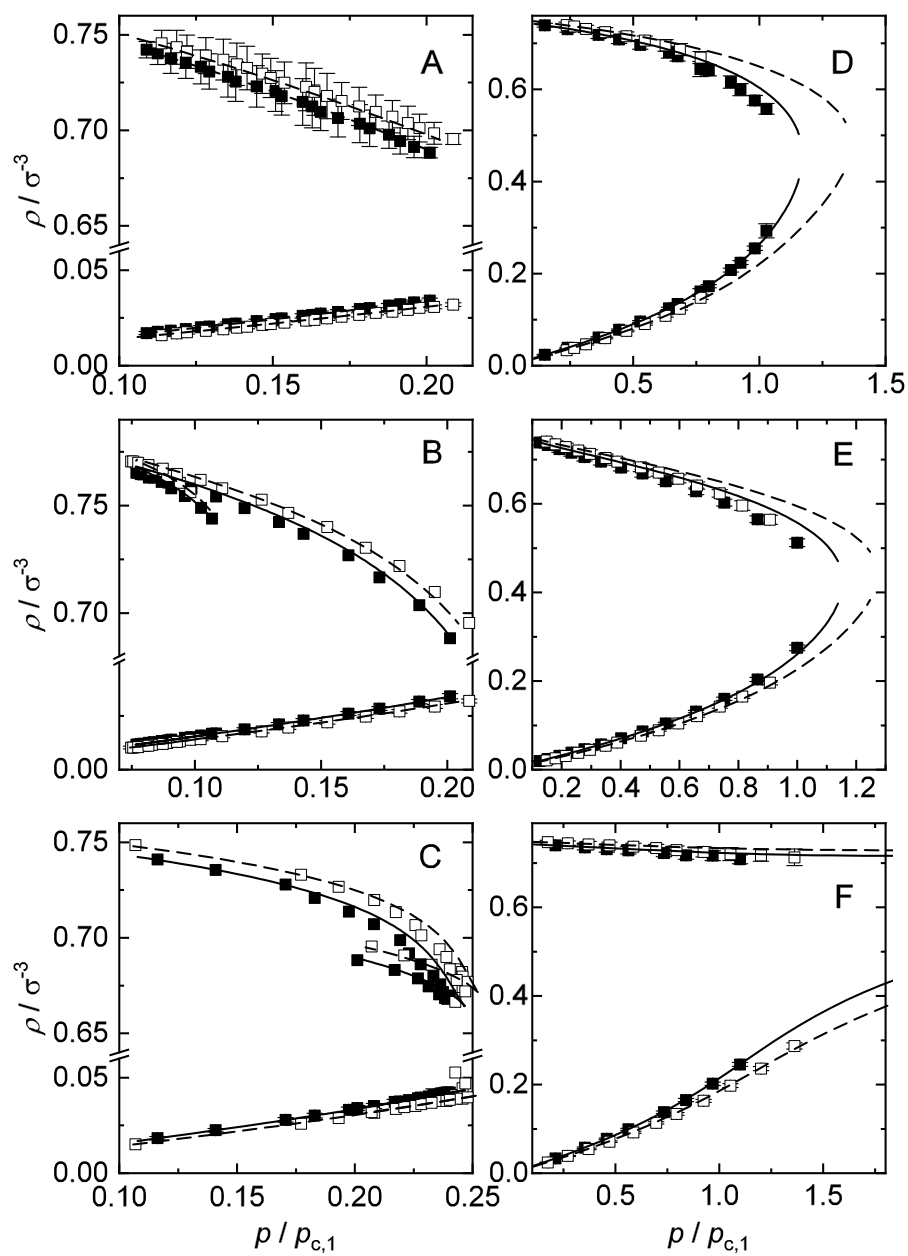

Figure 6. Saturated densities as a function of the pressure of the binary mixtures $\mathrm{A}, \mathrm{B}, \mathrm{C}, \mathrm{D}, \mathrm{E}$, and $\mathrm{F}$ at $T / T_{\mathrm{c}, 1}=0.7$, where $T_{\mathrm{c}, 1}$ is the critical temperature of the high-boiling component 1. Symbols are MD results, lines are EOS results. Black lines and black symbols indicate the LJ potential; dashed lines and open symbols indicate the LJTS potential. For the mixtures B and C, the MD error bars for the liquid density were omitted to avoid visual clutter. 

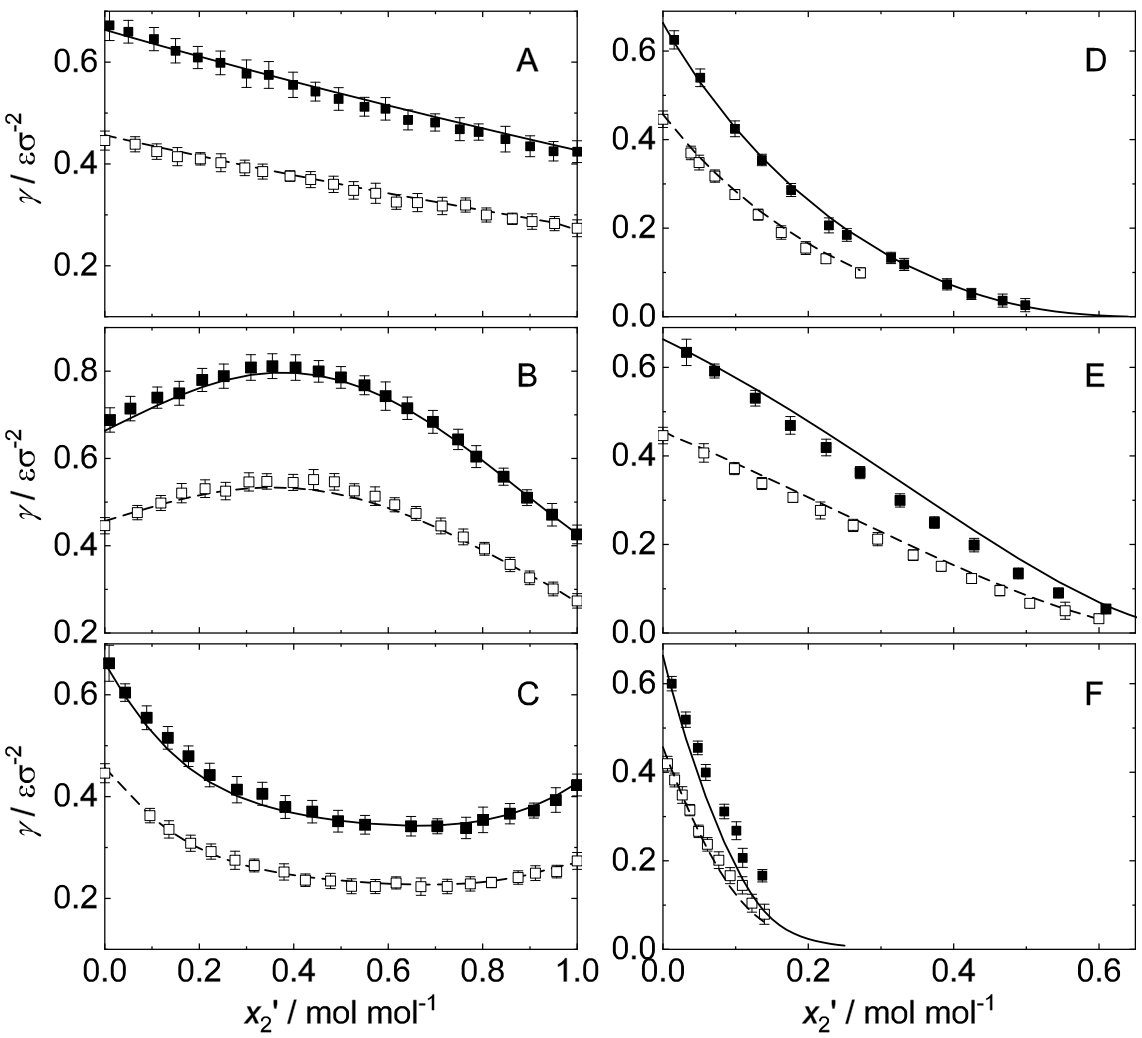

Figure 7. Surface tension $\gamma$ of the binary mixtures A, B, C, D, E, and F at $T / T_{\mathrm{c}, 1}=0.7$ as a function of the liquid phase composition $x_{2}^{\prime}$. Symbols are MD results, lines are EOS+DGT results. Black lines and black symbols indicate the LJ potential; dashed lines and open symbols indicate the LJTS potential.

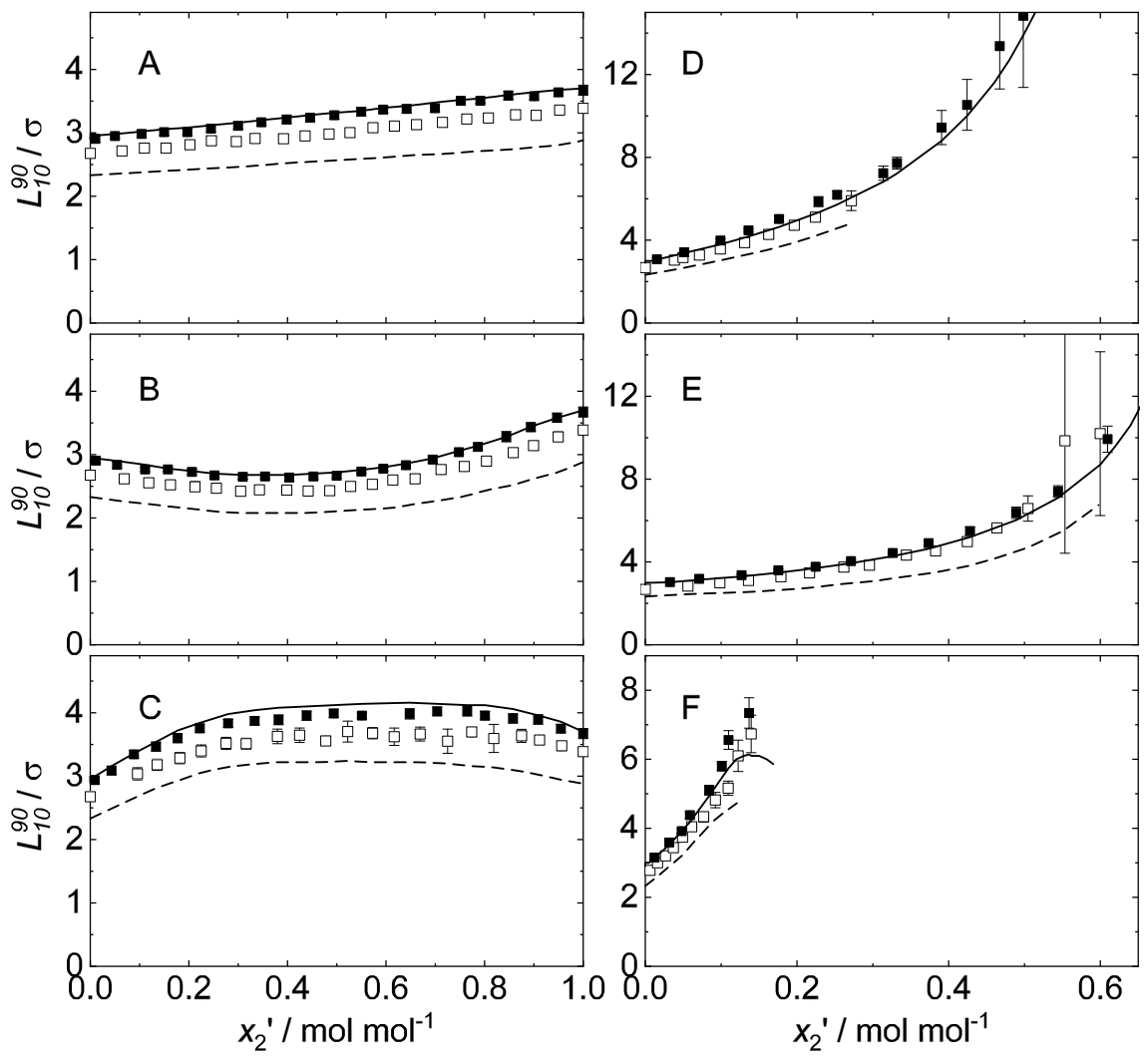

Figure 8. Interfacial thickness $L_{10}^{90}$ of the binary mixtures $\mathrm{A}, \mathrm{B}, \mathrm{C}, \mathrm{D}, \mathrm{E}$, and $\mathrm{F}$ at $T / T_{\mathrm{c}, 1}=0.7$ as a function of the liquid phase composition $x_{2}^{\prime}$. Symbols are MD results, lines are EOS+DGT results. Black lines and black symbols indicate LJ potential; dashed lines and open symbols indicate LJTS potential. 

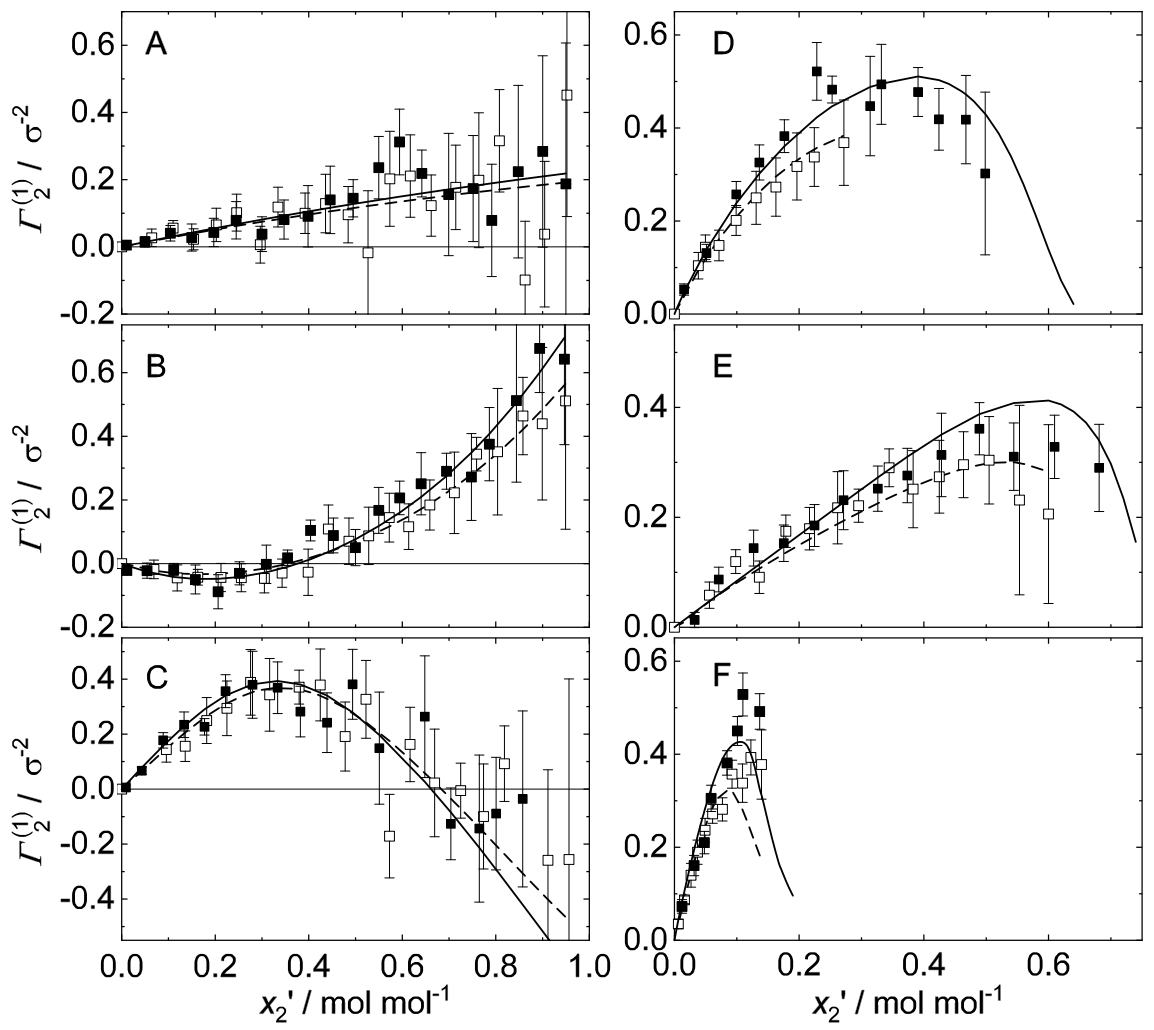

Figure 9. Relative adsorption $\Gamma_{2}^{(1)}$ of the low-boiling component of the binary mixtures $\mathrm{A}, \mathrm{B}, \mathrm{C}, \mathrm{D}, \mathrm{E}$, and $\mathrm{F}$ at $T / T_{\mathrm{c}, 1}=0.7$ as a function of the liquid phase composition $x_{2}^{\prime}$. Symbols are MD results, lines are EOS+DGT results. Black lines and black symbols indicate the LJ potential; dashed lines and open symbols indicate the LJTS potential.
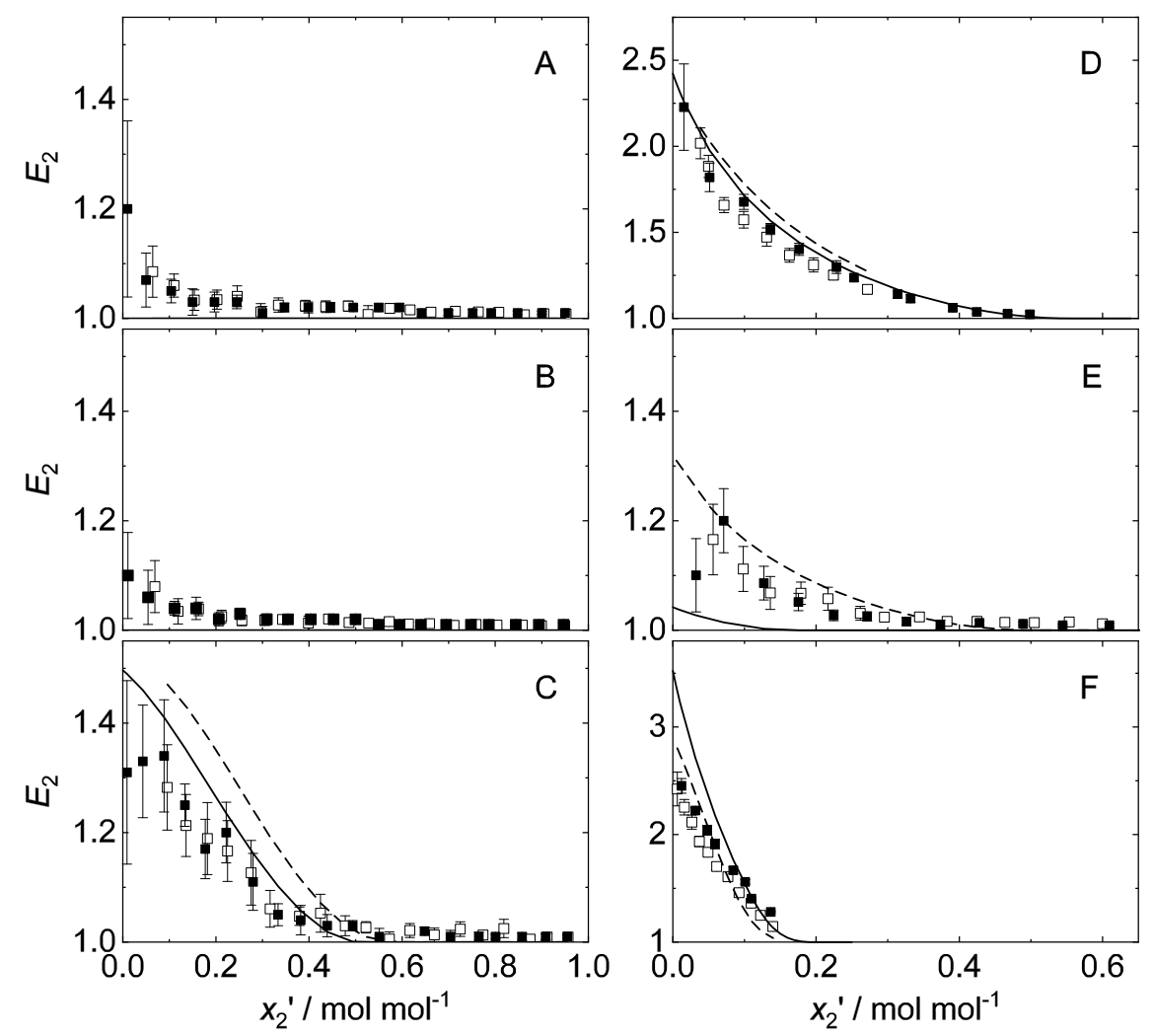

Figure 10. Enrichment of the low-boiling component $E_{2}$ at the interface of the binary mixtures A, B, C, D, E, and F at $T / T_{\mathrm{c}, 1}=0.7$ as a function of the liquid phase composition $x_{2}^{\prime}$. Symbols are MD results, lines are EOS+DGT results. Black lines and black symbols indicate the LJ potential; dashed lines and open symbols indicate the LJTS potential. In mixture A and B the enrichment obtained from EOS+DGT is $E_{2}=1.0$. 